\title{
Are neck flexion, neck rotation, and sitting at work risk factors for neck pain? Results of a prospective cohort study
}

G A M Ariëns, P M Bongers, $M$ Douwes, M C Miedema, W E Hoogendoorn, $G$ van der Wal, L M Bouter, W van Mechelen
TNO Work and Employment, PO Box $718,2130 \mathrm{AS}$ Hoofddorp, The Netherlands G A M Ariëns P M Bongers M Douwes M C Miedema W E Hoogendoorn

Institute for Research in Extramural Medicine, Faculty of Medicine, Vrije

Universiteit Amsterdam, The Netherlands G A M Ariëns W van Mechelen W E Hoogendoorn $\mathrm{G}$ van der Wal L M Bouter

Department of Social Medicine G A M Ariëns W van Mechelen W E Hoogendoorn $G$ van der Wal

Correspondence to: Dr G A M Ariëns g.ariens@arbeid.tno.nl

Accepted 3 November 2000

\begin{abstract}
Objective-To study the relation between neck pain and work related neck flexion, neck rotation, and sitting.

Methods-A prospective cohort study was performed with a follow up of 3 years among 1334 workers from 34 companies. Work related physical load was assessed by analysing objectively measured exposure data (video recordings) of neck flexion, neck rotation, and sitting posture. Neck pain was assessed by a questionnaire. Adjustments were made for various physical factors that were related or not related to work, psychosocial factors, and individual characteristics.

Results-A significant positive relation was found between the percentage of the working time in a sitting position and neck pain, implying an increased risk of neck pain for workers who were sitting for more than $95 \%$ of the working time (crude relative risk (RR) $2.01,95 \%$ confidence interval $(95 \% \mathrm{CI}) 1.04$ to 3.88 ; adjusted RR 2.34, $95 \%$ CI 1.05 to 5.21). A trend for a positive relation between neck flexion and neck pain was found, suggesting an increased risk of neck pain for people working with the neck at a minimum of $20^{\circ}$ of flexion for more than $70 \%$ of the working time (crude RR 2.01, 95\% CI 0.98 to 4.11; adjusted RR $1.63,95 \%$ CI 0.70 to 3.82 ). No clear relation was found between neck rotation and neck pain.

Conclusion-Sitting at work for more than $\mathbf{9 5 \%}$ of the working time seems to be a risk factor for neck pain and there is a trend for a positive relation between neck flexion and neck pain. No clear relation was found between neck rotation and neck pain.

(Occup Environ Med 2001;58:200-207)
\end{abstract}

Keywords: neck pain; physical risk factors; longitudinal cohort study

Neck pain is a major health problem in modern society. Prevalence data have shown that in a general population the 1 year prevalence of neck pain can be as high as $40 \%$, the prevalence for women being slightly higher. ${ }^{1}$ One year prevalences in occupational settings showed values varying between $6 \%$ and $76 \%$, also with higher values for female workers. ${ }^{1}$

Neck pain is assumed to be of multifactorial origin, implying that several risk factors can contribute to its development. Most studies which are reported in the literature focus on only one or a few risk factors. Moreover, most studies on risk factors for neck pain are of cross sectional design, which makes it difficult to formulate any conclusion about the temporal relation between risk factors and neck pain.

Among the many different putative risk factors for neck pain, work related physical risk factors may play a major part. Most studies on work related physical risk factors have collected information on exposure by questionnaires which are filled in by the workers. However, to validly measure the physical load at work, the duration and frequency of certain postures need to be assessed objectively.

Several literature reviews have specifically considered the work related physical risk factors for the development of neck pain..$^{2-5}$ However, due to differences in the design of these reviews, their conclusions are not fully consistent, although there seems to be consensus that potentially the main physical risk factors for neck pain are static postures and repetitive movements of the neck (neck flexion), static posture and repetitive or forceful movements of the arm, and a sitting posture at work.

The purpose of this study is to assess the longitudinal relation between neck pain and three physical risk factors related to work, neck flexion, neck rotation, and sitting posture. To our knowledge this is the first prospective cohort study in which this relation has been assessed on the basis of objectively measured exposure data, and in which the potential confounding effect of various psychosocial factors, physical factors not related to work, and individual characteristics has been taken into account.

\section{Methods}

DESIGN

In 1994, the study on musculoskeletal disorders, absenteeism, stress and health (SMASH), a large prospective cohort study with a follow up period of 3 years, was initiated among a working population. The main purpose of this study was to find the risk factors for musculoskeletal disorders, with a focus on back, neck, and shoulder disorders. About 1800 male and female workers from 34 companies participated in this study. The companies recruited were located throughout The Netherlands. Each company supplied 15-200 participants. In The Netherlands, all companies are connected to an occupational health service. The 
occupational health services gave information about companies possibly of interest to this study: companies not planning a major reorganisation or change in production within the next 3 years. Furthermore, to be included, the turnover rate of the workforce had to be less than $15 \%$. Companies from various industrial and service branches were included in the study, such as the metal industry, computer software industry, chemical industry, pharmaceutical industry, food industry, wood construction industry, insurance companies, childcare centres, hospitals, distribution companies, and bricklayers. This resulted in a study population of workers with various tasks and with a wide range of physical and mental workloads. Workers who were included had to have a more or less fixed workplace (to make video recordings at the workplace) and had to be able to read and write in the Dutch language (to fill in questionnaires).

Only those aspects of the methods that are relevant to the present study will be described in more detail. Extensive information on the study design and data collection can be found elsewhere. ${ }^{6}$

STUDY POPULATION

At the start of the study, $1789(87 \%)$ of the 2064 workers who were invited to participate in SMASH filled in the questionnaire at baseline. Furthermore, at baseline, workers had to meet the following five criteria.

(1) No other paid job for any substantial amount of time (18 workers were excluded).

(2) No work disability payment due to neck pain in the previous 12 months (three workers were excluded).

(3) Working for at least 20 hours a week.(11 workers were excluded).

(4) Working for at least 1 year in their current job (18 workers were excluded).

(5) No self reported regular or prolonged neck pain in the 12 months before baseline (405 workers were excluded).

After applying these selection criteria, 1334 workers were eligible for participation in the study.

ASSESSMENT OF EXPOSURE

At baseline, work related physical load was assessed by video recordings and force measurements at the workplace, and subsequent observation and analysis of these video recordings.

Research assistants who made the video recordings were trained to ascertain repeatable results and to minimise variability between observers. A standardised protocol was given to each research assistant, including an extensive description on how the video recording at the workplace should be made. Four 10 or 14 minute video recordings of each participant were taken randomly during a working day. All participants were assigned to groups of workers with similar tasks, based on inspection of the work on site. Video recordings of a quarter of the workers in each of these groups of workers were subsequently observed and analysed for relevant measures. To ease observations afterwards, markers were placed on different sites of the body of the worker. Video recordings were studied afterwards by a trained research assistant according to a standardised protocol. Video recordings were studied several times. Each time the research assistant concentrated on a different site of the body, and observations of that site only were made. Observations every 15 seconds were made of head inclination (in three categories: $0-20,20-45,>45^{\circ}$ from the neutral position), and head rotation (in two categories: $0-45,>45^{\circ}$ out of the neutral position). Continuous observations were made of working in a sitting position. Based on these observations and the analysis, the following four physical exposure variables were calculated.

(1) The percentage of the working time with the neck at a minimum of $20^{\circ}$ of flexion.

(2) The percentage of the working time with the neck at a minimum of $45^{\circ}$ of flexion.

(3) The percentage of the working time with the neck at a minimum of $45^{\circ}$ of rotation.

(4) The percentage of the working time in a sitting position.

For each group of workers, mean values were calculated for the physical exposure variables, based on the people observed within that group of workers. The mean value for each of the four physical exposure variables was then allocated to all people within that group.

ASSESSMENT OF NECK PAIN

At baseline, and annually during the follow up period, data on neck pain were collected with an adapted version of the Nordic questionnaire. ${ }^{7}$ On a four point scale (seldom or never; sometimes; regular; prolonged) workers had to rate the occurrence of neck pain in the previous 12 months. Cases of neck pain were considered to be workers who reported regular or prolonged neck pain during the previous 12 months on at least one of the three follow up measurements.

ASSESSMENT OF POTENTIAL CONFOUNDERS

Three confounding physical factors related to work were also derived from the video recordings (number of times lifting $25 \mathrm{~kg}$ or more in an 8 hour working day, the percentage of the working time with upper arm elevation of at least $60^{\circ}$, and the percentage of the working time making repeated movements with the hand or arm more than four times a minute). Furthermore, with a questionnaire, data were collected on other physical factors related to work (work at a video display terminal, working with the hands above shoulder level, working with vibrating tools, driving a vehicle, frequent flexion or rotation of the upper part of the body) and physical factors not related to work (prolonged sitting, work at a video display terminal, activities with the hands above shoulder level, exertion of force with hands or arms, activities in the same position for a long time, working with vibrating tools, frequent flexion or rotation of the upper part of the body, making repeated movements with the hands or arms many times a minute, driving a vehicle, 
frequency of participating in sports or performing heavy physical activities which cause sweating during the previous 4 months). ${ }^{89}$ The job content questionnaire was used to collect information on psychosocial factors related to work. ${ }^{10}$ The different items on this questionnaire were combined into the four dimensions proposed by Karasek et al-namely, quantitative job demands, decision latitude, support of supervisors, and support of coworkers. ${ }^{10}$ The precise calculation of these dimensions has been described by de Jonge et al. ${ }^{11}$ One other single item from the job content questionnaire was used to collect information on job security. Finally, three individual characteristics were taken into account as potential confounders: sex, age, and body mass index (BMI). Body height $(\mathrm{m})$ and body weight $(\mathrm{kg})$ were measured during a physical examination. If no measurements of body height and body weight were available from the physical examination, the relevant self reported measurements on the questionnaire were used to calculate BMI $\left(\mathrm{BMI}=\right.$ weight $/$ height $\left.^{2}\right)$.

ASSESSMENT OF THE ENDURANCE TIME OF THE NECK MUSCLES

The relation between physical load at work and neck pain may be influenced by the level of physical fitness of a worker, so that workers with a high level of physical fitness can be exposed to a higher physical load before problems with the musculoskeletal system will occur. From the physical examination at baseline, information was available on the endurance time of the neck muscles, measured by means of an adapted submaximal static strength test of these muscles. ${ }^{12-14}$ During this test, the endurance strength of the neck muscles was evaluated on the basis of the number of seconds the seated worker could keep the neck flexed at a $45^{\circ}$ angle, while wearing a helmet loaded with a weight of $2.5 \mathrm{~kg}$ for women and $5 \mathrm{~kg}$ for men. During this test the localised musculoskeletal discomfort method was applied to obtain a rating of the perceived feeling of discomfort in any part of the body. The localised musculoskeletal discomfort method consists of a 10 point Borg scale which indicates the amount of discomfort. Also, a body diagram was used to indicate the location of the discomfort. The test was concluded and the endurance time was noted if a worker reached a localised musculoskeletal discomfort score of 5 in the neck or upper back region, or if a subject reached an endurance time of 420 seconds, which was considered to be the maximum endurance time. Only those subjects without neck pain at the time of testing had to perform the submaximal strength test of the neck muscles.

\section{STATISTICS}

Cox's regression analysis, with a constant time variable, was used to model the relation between neck pain and the percentage of the working time with the neck at a minimum of $20^{\circ}$ of flexion, the percentage of the working time with the neck at a minimum of $45^{\circ}$ of flexion, the percentage of the working time with the neck at a minimum of $45^{\circ}$ of rotation, and the percentage of the working time in a sitting position, resulting in the calculation of a relative risk (RR) and its corresponding 95\% confidence interval $(95 \% \mathrm{CI}) .^{15} 16$

Firstly, univariate analyses of the relation between each of the physical exposure variables and neck pain were performed. Each physical exposure variable was divided into small categories of about $5 \%-10 \%$ of the working time. Categories showing similar effect estimates were combined into broader categories, resulting in the following categorisation of the four variables of physical exposure: the percentage of the working time with the neck at a minimum of $20^{\circ}$ of flexion in three categories $(<60 \%, 60-70 \%$, and $>70 \%$ of the working time), the percentage of the working time with the neck at a minimum of $45^{\circ}$ of flexion in three categories $(<5 \%, 5-10 \%$, and $>10 \%$ of the working time), the percentage of the working time with the neck at a minimum of $45^{\circ}$ of rotation in three categories $(<25 \%, 25-30 \%$, and $>30 \%$ of the working time), and the percentage of the working time in a sitting position in five categories $(<1 \%, 1 \%-50 \%$, $50 \%-75 \%, 75 \%-95 \%$, and $>95 \%$ of the working time). For all four physical exposure variables, the first category mentioned served as the reference category in all analyses. A comparable strategy was used to categorise the potential confounding psychosocial dimensions assessed by the questionnaire and the three potential confounding physical factors assessed by video recordings.

Univariate analyses were performed to test the relation between neck pain and all potential confounders. Variables associated with neck pain with $p>0.25$ were not considered to be likely confounders. ${ }^{17}$ For all potential confounders with univariate $\mathrm{p}<0.25$, the actual confounding effect on the estimated RR of each physical exposure variable of interest was examined. Therefore, the estimated RR for each physical exposure variable resulting from a bivariate analysis (physical exposure variable and confounder) was compared with the crude $R R$. If the change in $R R$ was in the region of $10 \%$ or higher, the potential confounder was considered to be a real confounder in this dataset. Age, sex, and the other three physical exposure variables were selected initially as confounders, and included in all multivariate analyses. Finally, in the last step of the analysis, a multivariate model was constructed for each physical exposure variable, in which all confounders assessed during the previous steps of the analysis were included.

To assess the influence of possible misclassification of physical exposure due to changes in work during the follow up period, which may result in an underestimation or overestimation of the RR, the multivariate analysis of each physical exposure variable, with adjustment for confounders, was repeated for those workers who experienced no major changes in their work during the follow up period, with the reason for change in work being other than neck pain $(n=686)$. 
Table 1 Descriptive information and the distribution of the four variables related to physical exposure at work calculated from the video recordings of the workers included in the analyses $(n=977)$

\begin{tabular}{|c|c|c|c|c|}
\hline Variable & Classification & $\begin{array}{l}\text { Distribution } \\
n(\%) \text { Men }\end{array}$ & $\begin{array}{l}\text { Distribution } \\
n(\%) \text { Women }\end{array}$ & $\begin{array}{l}\text { Distribution } \\
n(\%) \text { Total }\end{array}$ \\
\hline Sex & & $737(74.4)$ & $240(25.6)$ & $977(100)$ \\
\hline Age $(y)^{\star} \dagger$ & & $36.8(8.1)$ & $32.1(8.8)$ & $35.7(8.5)$ \\
\hline Nationality^ & Dutch & $721(97.8)$ & $228(95.0)$ & $949(97.1)$ \\
\hline Employment in current job $(y)^{\star} \dagger$ & & $10.5(8.1)$ & $6.7(5.1)$ & $9.6(7.7)$ \\
\hline Work $(\mathrm{h} / \text { week })^{\star} \dagger$ & & $39.2(3.4)$ & $35.3(6.5)$ & $38.2(4.7)$ \\
\hline \multirow[t]{3}{*}{ Working time with neck at $\geqslant 20^{\circ}$ of flexion $(\%)^{\star} \ddagger$} & $<60$ & $683(95.9)$ & $191(80.9)$ & $874(92.2)$ \\
\hline & $60-70$ & $23(3.2)$ & $22(9.3)$ & $45(4.7)$ \\
\hline & $>70$ & $6(0.8)$ & $23(9.7)$ & $29(3.1)$ \\
\hline \multirow[t]{3}{*}{ Working time with neck at $\geqslant 45^{\circ}$ of flexion $(\%)^{\star} \ddagger$} & $<5$ & $547(76.8)$ & $154(65.3)$ & $701(71.8)$ \\
\hline & $5-10$ & $135(19.0)$ & $38(16.1)$ & $173(17.7)$ \\
\hline & $>10$ & $30(4.2)$ & $44(18.6)$ & $74(7.6)$ \\
\hline \multirow[t]{3}{*}{ Working time with neck at $\geqslant 45^{\circ}$ of rotation $(\%) \ddagger$} & $<20$ & $609(85.5)$ & $211(89.4)$ & $820(86.5)$ \\
\hline & $20-30$ & $64(9.0)$ & $15(6.4)$ & $79(8.3)$ \\
\hline & $>30$ & $39(5.5)$ & $10(4.2)$ & $49(5.2)$ \\
\hline \multirow[t]{5}{*}{ Working time in a sitting position $(\%)^{\star} \ddagger$} & $<1$ & $209(29.4)$ & $18(7.6)$ & $227(23.9)$ \\
\hline & $1-50$ & $288(40.4)$ & $101(42.8)$ & $389(41.0)$ \\
\hline & $50-75$ & $23(3.2)$ & $22(9.2)$ & $45(4.7)$ \\
\hline & 75-95 & $152(20.6)$ & $69(29.2)$ & $221(23.3)$ \\
\hline & $>95$ & $40(5.6)$ & $26(11.0)$ & $66(7.0)$ \\
\hline
\end{tabular}

${ }^{\star} \mathrm{p}<0.05$ difference between men and women.

tMean (SD).

$\ddagger$ For 29 workers, data on the physical exposure variables were missing.

It may be expected that for workers with a relatively high endurance time of the neck muscles, the relation between neck flexion and neck pain would be less pronounced than for workers with a relatively low endurance time. To test this hypothesis, a stratified analysis of the relation between neck flexion and neck pain was performed, dividing the population into tertiles, based on the results of the static endurance strength test of the neck muscles.

\section{Results}

SELECTIVE LOSS TO FOLLOW UP

In total, 1334 subjects met the inclusion criteria. A total of 357 subjects $(26.8 \%)$ did not provide complete data on the occurrence of neck pain, and were therefore considered to be lost to follow up. No difference was found in the distributions of neck flexion and neck rotation between the groups, whereas in the group of workers who were lost to follow up, significantly fewer were working in a sitting position for a high percentage of the working time. The incidence of neck pain during the first year of follow up showed that there was no significant difference between the group of workers with complete follow up data and the group of workers for whom data on the incidence of neck pain were missing for the 2 nd or 3rd year of follow up.

Of the 977 subjects included in the analyses, 686 subjects $(70.2 \%)$ did not experience major changes in their work during the follow up period, with any reason for change in work being other than neck pain.

\section{DESCRIPTIVE INFORMATION ON THE STUDY}

POPULATION

Table 1 presents descriptive information (sex, age, nationality, years of employment in current job, and working hours a week), and the baseline distribution of the physical exposure variables for the 977 participants in the study. The mean (range) percentage of the working time was $36.3 \%(0 \%-79.1 \%), 4.0 \%$ $(0 \%-36.5 \%), 16.2 \%(1.8 \%-44.6 \%)$, and $38 \%$
$(0 \%-100 \%)$ for neck flexion more than $20^{\circ}$, neck flexion more than $45^{\circ}$, neck rotation more than $45^{\circ}$, and sitting, respectively.

A total of 56 workers $(5.7 \%)$ reported that they had neck pain during the 1 st year of follow up, and a total of 141 workers (14.4\%) reported that they had neck pain at least once during the total follow up period of 3 years. The putative confounders are listed in table 2 . Variables marked with an asterisk were univariately associated with neck pain $(\mathrm{p}<0.25)$.

The results of the univariate and multivariate analyses (RR and 95\% CIs) of the association of the four physical exposure variables and neck pain are presented in table 3.

\section{NECK FLEXION}

Compared with the reference category, workers with the neck at a minimum of $20^{\circ}$ of flexion for $60 \%-70 \%$ and for more than $70 \%$ of the working time had an increased crude RR for neck pain of 1.62 (95\% CI 0.85 to 3.09 ) and 2.01 (95\% CI 0.98 to 4.11 ), respectively. In the multivariate analysis these RRs were smaller (RR 1.21 for neck flexion for $60 \%-70 \%$ of the working time and RR 1.63 for neck flexion for more than $70 \%$ of the working time), also not significant. Unexpectedly, only slightly increased RRs were found for the percentage of the working time with the neck at a minimum of $45^{\circ}$ of flexion. The results of the univariate analysis of this variable showed an RR of 1.19 (95\% CI 0.78 to 1.82 ) for neck flexion for $5 \%-10 \%$ of the working time, whereas in the multivariate analysis the RR was 1.27 (95\% CI 0.81 to 1.97$)$.

\section{NECK ROTATION}

There was an RR of 1.33 (95\% CI 0.78 to 2.28) for neck pain from the univariate analysis for workers who had their neck at a minimum of $45^{\circ}$ of rotation for $25 \%-30 \%$ of the working time, and an RR of 1.40 (95\% CI 0.81 to 2.43 ) from the multivariate analysis. More than $30 \%$ of the working time with the neck at a minimum of $45^{\circ}$ of rotation gave no increase in $\mathrm{RR}$ for neck pain in either the univariate or the 
Table 2 Potential confounders of the association between physical exposure at work and neck pain

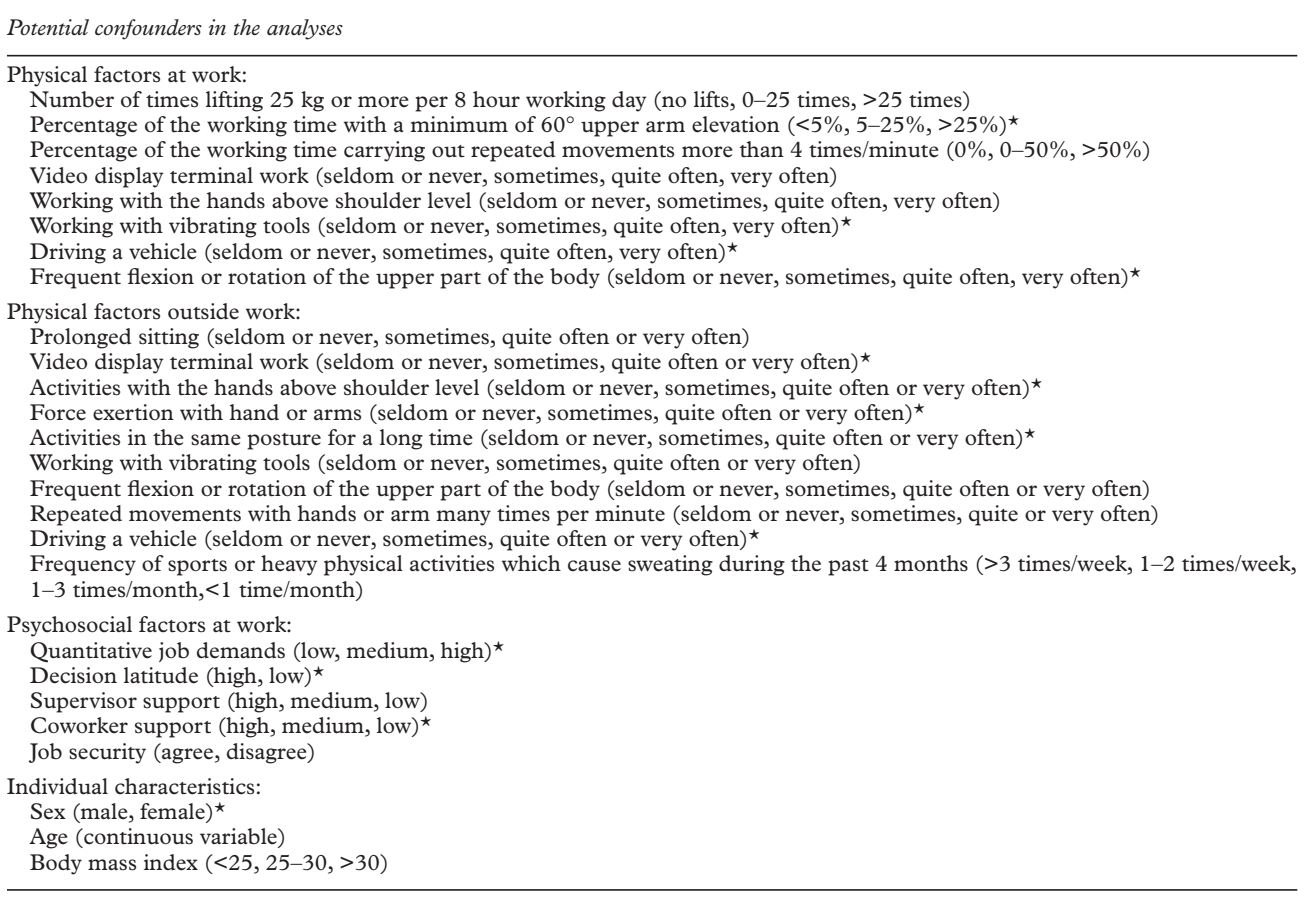

${ }^{\star} \mathrm{p}<0.25$, Univariate association with neck pain.

multivariate analysis (a crude RR of 0.86 and an adjusted $R R$ of 0.98 ).

SITTING POSTURE

Those who worked in a sitting position for more than $1 \%$ of the working time were at higher risk for neck pain than those who seldom worked in a sitting position (less than $1 \%$ of the working time). The univariate analysis showed an RR varying from 1.41 (95\% CI 0.88 to 2.27 ) for workers who were sitting for less than half of the working time to a significant RR of 2.01 (95\% CI 1.04 to 3.88 ) for workers who were sitting for more than 95\% of the working time. After adjustment for confounders, the RRs remained more or less the same, with the exception of the RR for sitting more than $95 \%$ of the working time, which increased to 2.34 (95\% CI 1.05 to 5.21$)$.

CHANGE IN PHYSICAL WORKLOAD DURING THE FOLLOW UP PERIOD

For the workers who had experienced no, or only minor changes in work during the follow

Table 3 Univariate and multivariate analysis of the association between variables of physical exposure at work and neck pain

\begin{tabular}{|c|c|c|c|c|c|c|}
\hline $\begin{array}{l}\text { Work related physical } \\
\text { exposures (\% of } \\
\text { working time) }\end{array}$ & $\begin{array}{l}\text { Confounders (as well as sex and age) included in the multivariate } \\
\text { analysis }\end{array}$ & $\begin{array}{l}\text { Neck } \\
\text { pain }\end{array}$ & $\begin{array}{l}\text { No neck } \\
\text { pain }\end{array}$ & $\begin{array}{l}\text { Crude RRt } \\
(95 \% \text { CI) }\end{array}$ & $\begin{array}{l}\text { Adjusted } R R \neq \\
(95 \% C I)\end{array}$ & $\begin{array}{l}\text { Subcohort, no } \\
\text { change in works } \\
\text { Adjusted RR } \\
(95 \% \text { CI) }\end{array}$ \\
\hline $\begin{array}{l}\text { Neck flexion } \geqslant 20^{\circ} \\
<60 \% \text { time } \\
60 \%-70 \% \text { time } \\
>70 \% \text { time }\end{array}$ & $\begin{array}{l}\text { Neck rotation (work) } \\
\text { Sitting posture (work) } \\
\text { Driving a vehicle (work) } \\
\text { Video display terminal work (leisure time) } \\
\text { Activities with hands above shoulder level (leisure time) } \\
\text { Activities in the same posture for a long time (leisure time) } \\
\text { Quantitative job demands }\end{array}$ & $\begin{array}{l}120 \\
10 \\
8\end{array}$ & $\begin{array}{l}754 \\
35 \\
21\end{array}$ & $\begin{array}{l}1.00 \\
1.62(0.85-3.09) \\
2.01(0.98-4.11)\end{array}$ & $\begin{array}{l}1.00 \\
1.21(0.58-2.53) \\
1.63(0.70-3.82)\end{array}$ & $\begin{array}{l}1.00 \\
1.76(0.78-3.94) \\
1.66(0.57-4.81)\end{array}$ \\
\hline $\begin{array}{l}\text { Neck flexion } \geqslant 45^{\circ} \\
<5 \% \text { time } \\
5 \%-10 \% \text { time } \\
>10 \% \text { time }\end{array}$ & $\begin{array}{l}\text { Neck rotation (work) } \\
\text { Sitting posture (work) } \\
\text { Driving a vehicle (leisure time) } \\
\text { Activities with hands above shoulder level (leisure time) } \\
\text { Force exertion with hands or arms (leisure time) }\end{array}$ & $\begin{array}{l}95 \\
28 \\
15\end{array}$ & $\begin{array}{l}606 \\
145 \\
59\end{array}$ & $\begin{array}{l}1.00 \\
1.19(0.78-1.82) \\
1.50(0.87-2.58)\end{array}$ & $\begin{array}{l}1.00 \\
1.27(0.81-1.97) \\
1.16(0.62-2.17)\end{array}$ & $\begin{array}{l}1.00 \\
1.16(0.66-2.04) \\
1.30(0.61-2.76)\end{array}$ \\
\hline $\begin{array}{l}\text { Neck rotation } \geqslant 45^{\circ} \\
<25 \% \text { time } \\
25 \%-30 \% \text { time } \\
>30 \% \text { time }\end{array}$ & $\begin{array}{l}\text { Neck flexion (work) } \\
\text { Sitting posture (work) }\end{array}$ & $\begin{array}{l}117 \\
15 \\
6\end{array}$ & $\begin{array}{l}703 \\
64 \\
43\end{array}$ & $\begin{array}{l}1.00 \\
1.33(0.78-2.28) \\
0.86(0.38-1.95)\end{array}$ & $\begin{array}{l}1.00 \\
1.40(0.81-2.43) \\
0.98(0.42-2.26)\end{array}$ & $\begin{array}{l}1.00 \\
1.25(0.61-2.55) \\
1.13(0.41-3.17)\end{array}$ \\
\hline $\begin{array}{l}\text { Sitting } \\
\quad<1 \% \text { time } \\
1 \%-50 \% \text { time } \\
50 \%-75 \% \text { time } \\
75 \%-95 \% \text { time } \\
>95 \% \text { time }\end{array}$ & $\begin{array}{l}\text { Driving a vehicle (work) } \\
\text { Frequent flexion or rotation of the upper part of the body (work) } \\
\text { Neck flexion (work) } \\
\text { Neck rotation (work) } \\
\text { Force exertion with hands or arms (leisure time) } \\
\text { Driving a vehicle (leisure time) } \\
\text { Quantitative job demands } \\
\text { Decision latitude }\end{array}$ & $\begin{array}{l}24 \\
58 \\
8 \\
34 \\
14\end{array}$ & $\begin{array}{l}203 \\
331 \\
37 \\
187 \\
52\end{array}$ & $\begin{array}{l}1.00 \\
1.41(0.88-2.27) \\
1.68(0.76-3.74) \\
1.46(0.86-2.45) \\
2.01(1.04-3.88)^{\star}\end{array}$ & $\begin{array}{l}1.00 \\
1.25(0.75-2.09) \\
1.43(0.59-3.50) \\
1.29(0.71-2.37) \\
2.34(1.05-5.21)^{\star}\end{array}$ & $\begin{array}{l}1.00 \\
1.79(0.86-3.74) \\
1.85(0.56-6.11) \\
1.58(0.68-3.63) \\
3.28(1.22-8.81)^{\star}\end{array}$ \\
\hline
\end{tabular}

${ }^{\star} \mathrm{p}<0.05$.

+Univariate Cox's regression analysis.

$\ddagger$ Multivariate Cox’s regression analysis.

$§ M u l t i v a r i a t e$ Cox's regression analysis, workers who experienced no major changes in work during the follow up period, for a reason other than neck pain ( $\mathrm{n}=686$ ). 
Table 4 Univariate analysis of the association between neck flexion at work and neck pain for workers with a low, medium, and high endurance time of the neck muscles

\begin{tabular}{llll}
\hline $\begin{array}{l}\text { Variable } \\
\text { (\% of working time) }\end{array}$ & $\begin{array}{l}\text { Low endurance time } \\
\text { Crude RR }(95 \% \text { CI }) t\end{array}$ & $\begin{array}{l}\text { Medium endurance time } \\
\text { Crude RR }(95 \% \text { CI })\end{array}$ & $\begin{array}{l}\text { High endurance time } \\
\text { Crude RR }(95 \% \text { CI })\end{array}$ \\
\hline $\begin{array}{l}\text { Neck flexion } \geqslant 20^{\circ}: \\
\quad<60 \% \text { time }\end{array}$ & 1.00 & 1.00 & 1.00 \\
$\quad>60 \%$ time & $2.50^{\star}(1.11$ to 5.61$)$ & $1.32(0.52$ to 3.35$)$ & $1.11(0.34$ to 3.65$)$ \\
$\begin{array}{l}\text { Neck flexion } \geqslant 45^{\circ}: \\
\quad<5 \% \text { time }\end{array}$ & 1.00 & 1.00 & 1.00 \\
$\quad>5 \%$ time & $1.89^{\star}(1.02$ to 3.52$)$ & $1.08(0.57$ to 2.05$)$ & $0.84(0.38$ to 1.86$)$ \\
\hline & & & \\
&
\end{tabular}

up period $(n=686)$ the multivariate analyses of the four physical exposure variables of interest showed, in general, a slightly higher estimated RR for neck pain for all physical exposure variables (last column of table 3), with the only significant RR being the one for working in a sitting position for more than $95 \%$ of the working time (RR 3.28, 95\% CI 1.22 to 8.81 ).

ENDURANCE TIME OF THE NECK MUSCLES Increasing risks for neck pain due to neck flexion were found with decreasing endurance times (table 4). However, the interaction term between neck flexion and endurance time in the multivariate analysis was not significant. Workers with the lowest endurance time (the lowest tertile) showed a significant increase in RR for neck pain with the percentage of the working time when the neck was at a minimum of $20^{\circ}$ of flexion and with the percentage of the working time when the neck was at a minimum of $45^{\circ}$ of flexion. For the other two tertiles the RRs for neck pain were smaller, and not significant (table 4).

\section{Discussion}

The purpose of this study was to identify the longitudinal relation between physical load related to work and neck pain. To our knowledge this is the first prospective cohort study in which this relation has been assessed on the basis of objectively measured exposure data, and in which the potential confounding effect of various important psychosocial factors, physical factors not related to work, and individual characteristics was taken into account.

A trend for a positive relation between neck flexion and neck pain was found, although not significant, suggesting an increased risk of neck pain for those who spent a high percentage of the working time $(>70 \%)$ with the neck at a minimum of $20^{\circ}$ of flexion. Working with the neck at a minimum of $20^{\circ}$ of flexion for $25 \%$ $50 \%$ or $50 \%-60 \%$ of the working time showed no increased RR for neck pain. For this reason, the analysis of the neck at a minimum of $20^{\circ}$ of flexion was concentrated on percentages higher than $60 \%$ of the working time. Unexpectedly, the RRs for neck pain were lower for the percentage of the working time with the neck at a minimum of $45^{\circ}$ of flexion. Other published studies reported results of the relation between neck flexion and neck pain, with odds ratios ranging from 1.7 to $3.4 .{ }^{18-20}$ By contrast with the present study, these studies were of a cross sectional design and used a questionnaire for the assessment of neck flexion.
No clear relation was found between neck rotation and neck pain in the scientific literature. The results reported for neck rotation are not consistent. For example, Dartiques et al reported a positive effect (odds ratio of $2.4,95 \%$ CI 1.5 to 3.8 ) of cervical spine rotation on self reported neck pain, whereas Musson et al reported that the association between neck rotation and neck pain was not significant. ${ }^{18} 21$

In the present study, the power to investigate prolonged neck rotation was limited, as only a few of the workers had their neck rotated by more than $45^{\circ}$ for $30 \%$ of the working time or more. Setting the limit for prolonged neck rotation at an even higher level (for example more than $50 \%$ of the working time) was impossible due to this small number of workers.

A significant positive relation was found between sitting posture and neck pain. For workers who sat for more than $95 \%$ of the working time the risk of neck pain was twice as high as for workers who hardly ever worked in a sitting position. The results of the present study confirmed previous findings. Skov et al also investigated the effect of working time in a sitting position on self reported neck pain. ${ }^{22}$ They found that the odds ratios for neck pain increased with the time spent working in a sitting position (an odds ratio of 2.68 for a quarter of the working time in a sitting position, an odds ratio of 1.92 for half of the working time in a sitting position, an odds ratio of 2.18 for three quarters of the working time in a sitting position, and an odds ratio of 2.80 for all of the working time in a sitting position), suggesting a clear relation between sitting posture and neck pain. Kamwendo et al reported an odds ratio of 1.49 (95\% CI 0.86 to 2.61 ) for the relation between sitting for more than 5 hours a day and self reported neck pain. ${ }^{23}$ Both of these studies had a cross sectional design and used data from questionnaires to assess exposure.

A plausible mechanism for the strong relation between prolonged sitting and neck pain which is found in this study is the static aspect of this exposure. Working in a sitting position will lead to a continuous static load on the neck muscles, especially if the design of the workplace is not suitable for the worker. Static loading of the neck muscles will induce biomechanical strain-for example, an increased muscle tone-which may in the long term lead to the development of neck pain.

The possible interrelations between work related neck flexion, neck rotation, and sitting could have had an important influence on the results found in this study. Untangling the independent effect of these variables is difficult. By checking the correlations between the physical exposure variables, the interrelations between the variables were verified. All Pearson correlation coefficients were below 0.3 . These relatively low correlation coefficients may imply a relatively small influence of possible interrelations between the physical exposure variables on the results found in this study, so the precise magnitude of the independent effects should be interpreted with caution. 
When the multivariate analyses were performed for workers who had experienced no changes, or only minor changes in work during the follow up period $(n=686)$, slightly higher RRs for neck pain were found for neck flexion and sitting posture. However, the overall conclusions based on the results of both analyses remained the same. It can therefore be concluded that change in work during the follow up period had only a minor influence on the magnitude of the RRs found for the relation between the physical exposure variables and neck pain.

Workers with a relatively long endurance time of the neck muscles had a less pronounced relation between neck flexion and neck pain than did workers with a relatively short endurance time. A significant increase in RRs for neck pain was found for workers with the lowest endurance times measured by the static strength test of the neck muscles, whereas for the workers with medium and high endurance times, there was no significant increase in risk of neck pain. These results suggest that working with a flexed neck is a real problem for workers with a low endurance time in the neck muscles.

Instead of questionnaires, which were used in most reported studies, the present study used a standardised method to measure the physical load at the workplace. However, additional data on neck flexion, neck rotation, and sitting posture were collected by a questionnaire. To assess whether these two methods of data collection would result in comparable conclusions about the relations between neck flexion, neck rotation, sitting posture, and neck pain, the data obtained from the questionnaire were also related to neck pain. Similar results were found for the relation between neck pain and neck flexion (often working with a flexed neck) and neck rotation (often working with a rotated neck). However, for prolonged sitting, no increased risk of neck pain could be detected from the data obtained from the questionnaire (data not shown). Thus, the conclusion that prolonged neck flexion is related to neck pain is supported by the results based on the data obtained from the questionnaire. However, this is not the case for the relation between working in a sitting position and neck pain.

LIMITATIONS OF THIS STUDY

Bias due to loss to follow up may have occurred in the present study. Out of the initial 1334 workers, 977 had complete follow up data. Three hunderd and fifty seven workers $(26.8 \%)$ were considered to be lost to follow up. Of these 357 workers, 211 workers did provide data at the first follow up, but were lost at the second or third follow up measurement. Eighteen $(8.5 \%)$ of these 211 workers reported neck pain at the first follow up. The workers who were lost to follow up had a lower level of education. Whether this difference in level of education has influenced the relation between the physical exposure data and the occurrence of neck pain during the follow up period cannot be investigated, because no data are available on the cumulative incidence of neck pain during the follow up period for the workers who were lost to follow up.

With the selection of subjects without regular or prolonged neck pain in the 12 months before baseline, we have eliminated a strong confounding effect of recent previous neck pain. However, it is still possible that neck pain more than 1 year before baseline may have had its influence on the relation between the physical variables related to work and the occurrence of neck pain during the follow up period.

Four video recordings of 14 minutes were made of each worker who participated in the baseline measurements. Due to time restrictions, it was impossible to observe all video recordings of all workers in this study. Therefore all participants were assigned to groups of workers with similar tasks, based on inspection of the worksite. The research assistant who made the video recordings classified the workers into homogeneous groups. The division of workers into groups was not based on job title, but on inspection at the workplace.

Unfortunately, no information was available on the reliability of video observations between the raters. As other studies reported considerable problems with reproducibility of ratings of posture based on video recordings, the results of the present study should be interpreted with caution.

Non-differential misclassification of workers in exposure categories could have occurred in the present study. The size of this misclassification is unknown, however, it is the same for workers with and without neck pain. The effect of this non-differential misclassification can be an underestimation of the effect, because it tends to bias the effect estimate towards the null value. ${ }^{24}$ Unfortunately, misclassification for the four exposure variables was not studied. However, some information is available for another important physical exposure variable - namely trunk flexion. Results of prelimary analyses showed that for trunk flexion the grouping of workers resulted in homogeneous groups with relatively little variance within groups and large variance between groups. Other ways of grouping - for example, on the basis of job titles or similar function-showed higher variances within groups, but these were still smaller than the variance between groups. As trunk flexion was assessed in an identical way as the four physical exposure variables under study here, it is assumed that the variance within and between groups and the attenuation of the risk estimates are of comparable size.

Video recordings of a single worker were made on a single day. The variability within a person may be underestimated due to this, because variability in exposure over days, weeks, or seasons are ignored. However, measurements of physical exposure for a single worker on separate days, in different weeks or different seasons was, for practical reasons, impossible.

According to the scientific literature static loading of the neck muscles is an important risk 
factor for neck pain. ${ }^{3}$ Unfortunately, our findings do not consider static loading of the neck muscles. In a pilot study it turned out to be impossible to assess neck flexion and neck rotation continuously. We had to restrict our measurements of neck flexion and neck rotation to observations from the video recordings every 15 seconds. We were therefore not able to estimate whether workers were exposed to neck flexion or neck rotation continuously, or for short periods.

\section{Conclusions}

(1) There is a significant positive association between prolonged sitting at work and neck pain, implying that there is an increased risk of neck pain for people who are working almost all day in a sitting position (more than $95 \%$ of the working time). Due to the prospective study design and thorough adjustment for confounding, it can be concluded that this relation between sitting posture and neck pain is probably a causal relation.

(2) There is a positive trend for an association between neck pain and neck flexion at work, although not significant, suggesting that there is an increased risk of neck pain for people who are working with the neck flexed more than $20^{\circ}$ for a major part of their working day. A low endurance time of the neck muscles seems to play an important part in the development of neck pain due to neck flexion at work.

(3) No clear relation was found between neck rotation at work and neck pain.

(4) Based on the results of this study, prevention of neck pain should focus on the reduction of time spent working in a sitting position and the promotion of more dynamic working postures.

This study was financially supported by the Dutch Ministry of Social Affairs and Employment, the Dutch Ministry of Public Health, Welfare and Sports, and the Dutch National Institute of Social Security.

1 Ariëns GAM, Borghouts JAJ, Koes BW. Neck pain. In: Crombie IK, ed. The epidemiology of pain. Seattle, WA: IASP Press, 1999:235-55.

2 Kuorinka I, Forcier L, eds. Work related musculoskeletal disorders (WMSD): a reference book for prevention. London: Taylor and Francis, 1995:17-137.

3 Bernard BP, ed. Musculoskeletal disorders (MSDs) and workplace factors. Cincinnati $(\mathrm{OH})$ : United States Department place factors. Cincinnati (OH): United
of Health and Human Services, 1997.
4 Stock SR. Workplace ergonomic factors and the development of musculoskeletal disorders of the neck and upper imb: a meta-analysis. Am f Ind Med 1991;19:87-107.

5 Ariëns GAM, Van Mechelen W, Bongers PM, et al. Physical risk factors for neck pain. Scand $\mathcal{f}$ Work Environ Health 2000;26:7-19.

6 Bongers PM, Miedema MC, Douwes M, et al. Longitudinal study on low back, neck, and shoulder symptoms. Part 1: research design and data collection. (In Dutch.) Hoofddorp, The Netherlands: TNO Arbeid, 2000.

7 Kuorinka I, Jonsson B, Kilbom Å, et al. Standardised Nordic questionnaires for the analysis of musculoskeletal symptoms. Applied Ergonomics 1987;18:233-7.

8 Hildebrandt VH, Douwes M. Physical load and work: questionnaire on musculoskeletal load and health complaints (Lichamelijke belasting en arbeid: vragenlijst bewegingsapparaat).Voorburg: Ministerie van Sociale Zaken bewegingsapparaat).Voorburg: Ministerie van Sociale Zaken 1991. (Studies S122-3.)

9 Godin G, Jobin J, Bouillon J. Assessment of leisure time exercise behaviour by self report: a concurrent validity study. Can F Public Health 1986;77:359-62.

10 Karasek RA, Pieper CF, Schwartz JE. Job content questionnaire and user's guide: revision 1. Los Angeles: USCLA, 1985.

11 De Jonge J, Reuvers MMEN, Houtman ILD, et al. Linear and non-linear relations between psychosocial job characteristics, subjective outcomes and sickness absence: baseline results from SMASH. F Occup Health Psychol 2000;5:256-68

12 Hagberg $M$, Hogstedt C. Stockholmundersokningen 1 MUSIC-Books. Stockholm: Karolinskan Sjukhuset, 1991: 290-302, 328-33.

13 Theorell T, Schüldt C, Ekholm J, et al. Physical strength and endurance in relation to perceived psychosocial work environment, sleep disturbance and coping strategies in men. ronment, sleep disturbance and cop

14 Ekholm J, Schüldt K, Harms-Ringdahl K, et al. Normative data of muscular endurance in cervical spine extensors, shoulder flexors, and wrist extensors: data from a randomly selected population [abstract]. Rome, Italy: Meeting of the European Society of Biomechanics, 1992:289-90.

15 Lee J. Odds ratio or relative risk for cross-sectional data? Int 7 Epidemiol 1994;23:201-3.

16 Thompson ML, Myers JE, Kriebel D. Prevalence odds ratio or prevalence ratio in the analysis of cross sectional data: what is to be done? Occup Environ Med 1998;55:272-7.

17 Hosmer DW, Lemeshow S. Model-building strategies and methods for logistic regression. In: Hosmer DW, Lemeshow S, eds.Applied logistic regression. New York: John Wiley, 1998;82-134.

18 Dartiques JF, Henry P, Puymirat E, et al. Prevalence and risk factors of recurrent cervical pain syndrome in a working population. Neuroepidemiology 1988;7:99-105.

19 Kilbom Å, Persson J, Jonsson BG. Disorders of the cervicobrachial region among female workers in the electronics industry. International fournal of Industrial Ergonomics 1986;1:37-47.

20 Ignatius YTS, Yee TY, Yan LT. Self-reported musculoskeletal problems amongst typists and possible risk factors. f Hum Ergol (Tokyo) 1993;22:83-93.

21 Musson Y, Burdorf A, Van Drimmelen D. Exposure to shock and vibration and symptoms in workers using impact power tools. Ann Occup Hyg 1989;33:85-96.

22 Skov T, Borg V, Ørhede E. Psychosocial and physical risk factors for musculoskeletal disorders of the neck, shoulders, and lower back in salespeople. Occup Environ Med 1996;53:351-6.

23 Kamwendo K, Linton SJ, Moritz U. Neck and shoulder disorders in medical secretaries. Scand $\mathcal{f}$ Rehabil Med 1991;23:127-33.

24 Copeland KT, Checkoway H, McMichael AJ, et al. Bias due to misclassification in the estimation of relative risk. Am $\mathcal{F}$ Epidemiol 1977;105:488-95. 